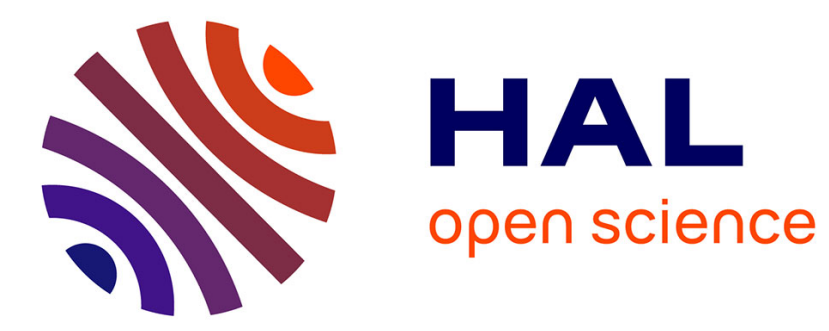

\title{
Dispersion analysis in ballasted railway tracks and Anderson localization in granular media
}

\author{
L de Abreu Corrêa, R. Cottereau, B. Faure
}

\section{To cite this version:}

L de Abreu Corrêa, R. Cottereau, B. Faure. Dispersion analysis in ballasted railway tracks and Anderson localization in granular media. Journal of Sound and Vibration, 2020, 465, pp.115010. 10.1016/j.jsv.2019.115010 . hal-02566875

\section{HAL Id: hal-02566875 \\ https://hal.science/hal-02566875}

Submitted on 14 May 2020

HAL is a multi-disciplinary open access archive for the deposit and dissemination of scientific research documents, whether they are published or not. The documents may come from teaching and research institutions in France or abroad, or from public or private research centers.
L'archive ouverte pluridisciplinaire HAL, est destinée au dépôt et à la diffusion de documents scientifiques de niveau recherche, publiés ou non, émanant des établissements d'enseignement et de recherche français ou étrangers, des laboratoires publics ou privés. 


\title{
Dispersion analysis in ballasted railway tracks and Anderson localization in granular media
}

\author{
L. de Abreu Corrêa ${ }^{a}$, R. Cottereau ${ }^{a, b, *}$, B. Faure ${ }^{c}$ \\ ${ }^{a}$ MSSMat, CNRS, CentraleSupélec, Université Paris-Saclay, France \\ ${ }^{b}$ Aix Marseille Univ, CNRS, Centrale Marseille, LMA UMR7031, France \\ ${ }^{c}$ SNCF, Innovation \& Research Dept., France
}

\begin{abstract}
This paper analyzes the dynamic behavior of a randomly-heterogeneous continuum model of a ballasted railway track through the numerical construction of dispersion curves along the axis of the track. At intermediate frequencies $(50-100 \mathrm{~Hz})$, the dispersion curves display a strong decrease of the group velocity. At higher frequencies (above $100 \mathrm{~Hz}$ ), the wave are strongly localized and cannot propagate along the track. This behavior is coherent with experimental observations reported in the literature on ballasted railway tracks and other granular media. Additional simulations are performed to prove that the localization is not a consequence of the geometry of the track or the impedance mismatch between the ballast and the underlying soil, but is indeed Anderson localization due to the heterogeneity of the ballast.

Keywords: Ballasted railway tracks, Granular media, Heterogeneous media, Random media, Anderson localization
\end{abstract}

2010 MSC: 00-01, 99-00

\section{Introduction}

The ballast is the uppermost layer of the railway track superstructure, on which the sleepers stand. It is made of coarse crushed stones, whose size distribution lies in the range $25-50 \mathrm{~mm}$. This ballast layer plays an important role in the transmission and

\footnotetext{
* Corresponding author

Email address: cottereau@lma.cnrs-mrs.fr (R. Cottereau)
}

Preprint submitted to Journal of Sound and Vibration

May 14, 2020 
5 repartition of static and dynamic train loads, in the absorption of mechanical and acoustical vibrations, and in the drainage of rain water [1]. The noise of the passage of a train and its vibration impact on the environment can be important for all types of trains: heavy freight coaches, whose low frequency impact in the soil carries far away; highspeed trains, that generate high amplitude excitations; and even tramways (although more rarely on ballasted tracks), which stand very close to surrounding buildings. This vibration impact may sometimes be strong enough to force the train companies to locally reduce the velocity of the passing trains or force the companies change the track. The modeling of the ballast aims at improving the understanding of the mechanical behavior of the track system and providing clues to mitigate this issue [2, 3]. It represents a challenging task because granular materials exhibit various distinct behaviors (such as transition between fluid-like and solid-like behavior) depending on the applied stresses and strains.

Classical numerical models of granular media include discrete models and continuum homogeneous models. Unfortunately, they are both inappropriate for dynamic analyses of ballasted railway tracks. Indeed, the latter produce vibration levels away from the track that are much larger than experimentally measured, while the former are too numerically expensive for such applications. Recently, a randomly-fluctuating heterogeneous continuum model of the ballast was proposed [4]. This model is based on continuum mechanics, so that it is parameterized with Lamé parameters and den25 sity, which are constant within the domain in classical models. In the proposed model, however, they fluctuate at the scale of the grains, which allows to recover the strong heterogeneity of the stress field, characteristic of granular media and very influential on its dynamic behavior [5, 4]. Finally, the fluctuations of the mechanical parameters are modeled as random fields and parameterized with statistical parameters (average, variance, correlation length). More details on that model will be recalled in Section 3.1 The model was shown to be coherent with previously unexplained experimental observations, and to reproduce stress distributions obtained by discrete simulation of assemblies of grains. Being continuum-mechanics based, it is amenable to very efficient numerical approximation of large scale problems involving ballasted railway tracks.

35 The main objective of this paper is to analyze precisely the dynamical behavior of this 
randomly fluctuating model and confirm its validity for ballasted railway tracks. This analysis will be performed through the numerical construction of dispersion curves for that model of the ballast.

Dispersion curves are among the most classical tools used in the analysis of wave 40 propagation. These curves link the values of pairs of wave numbers and frequencies $(k, \omega)$ for which a plane wave can actually propagate in the medium. These simple 2D diagrams allow for a quick understanding of the properties of the wave propagation medium, independently of the source that may excite it. For a given excitation frequency, the curves chart which waves will propagate in the medium considered, and with what wave number. They also allow to understand the way a given wave will evolve with frequency, the so-called dispersion characteristics of that wave. In some rare cases, the dispersion curves can be constructed analytically. For instance, in linear elastic isotropic infinite media, the dispersion curves are two straight lines, for the pressure and shear waves, respectively. For half-spaces and thin media, respectively, the dispersion curves for Rayleigh waves [6] and Lamb waves [7] can likewise be constructed analytically. In three dimensions, the wave number is a vector, with each coordinate describing propagation along a given direction of space, and the dispersion curve is actually a volume in a 4D space. To simplify the presentation, this paper concentrates on the construction of the dispersion relation for wave numbers along the axis of the track, hence exposing classical plot, with frequency on one axis, and a scalar wave number along the other axis.

In granular media, among which the ballast, wave propagation phenomena are more difficult to analyze [8, 9]. For instance, dispersion curves can be constructed experimentally for waves at the surface of unconsolidated granular media in dry condition [10,11] and present a very complex behavior. Waves in granular media are carried by complex force chains, which create a heterogeneous and non-isotropic behavior, as observed with photo-elastic imaging [12, 13]. Also, the contact chains are easily broken, deformed and rearranged [14], creating a nonlinear behavior of the medium. Even in the purely linear case, granular media are also dispersive, which is difficult to capture through classical continuum models [15]. Through the construction of numerical dispersion curves for the randomly-fluctuating continuum model of the ballast, this 
paper will reproduce some of the features of these experimental dispersion curves.

After the presentation of a general methodology for the numerical construction of dispersion curves in Section 2, this construction will be the focus of Section 3 . There, it will be shown that high-frequency waves (above $100 \mathrm{~Hz}$ ) cannot propagate in this ballasted railway track. This will be analyzed as Anderson localization [16, 17], which is a very specific physical phenomenon that appears in disordered media, such as granular packings. When localized, the waves cannot propagate over long distances because of the heterogeneity of the parameters and remain in the vicinity of the source. Although more classical in quantum systems, experimental evidence of Anderson localization exist for classical waves [18, 19, 20], and in particular in granular media [21]. To make sure that the localization observed in Section 3 is not a simple wave guide effect, or a consequence of the impedance mismatch between the ballasted railway track and the underlying soil, additional simulations will be performed in Section 4 .

\section{8о 2. Construction of the dispersion curve using numerical solutions in time-space}

In this section, we present a methodology to obtain dispersion curves using numerical solutions of the wave equation in space-time.

\subsection{Definition of dispersion curves}

Let us consider the equilibrium equation, neglecting the influence of the gravity forces, states:

$$
\nabla \cdot \sigma+\mathbf{f}=\rho \ddot{\mathbf{u}}
$$

where $\mathbf{u}(\mathbf{x}, t)$ is the displacement field, $\sigma$ represents the stress tensor, $\varepsilon=(\nabla \mathbf{u}+$ $\left.\nabla \mathbf{u}^{T}\right) / 2$ is the strain, $\mathbf{f}$ represents external volume forces, and $\nabla$ is the differential operator. We assume that the material is heterogeneous, linear, and isotropic, so that $\sigma=\lambda(\mathbf{x}) \operatorname{Tr} \varepsilon \mathbb{I}+2 \mu(\mathbf{x}) \varepsilon$ with $\lambda(\mathbf{x})$ and $\mu(\mathbf{x})$ the Lamé coefficients, and $\mathbb{I}$ the identity second-order tensor. The density is denoted $\rho(\mathbf{x})$. The parameters are related to the wave velocities through $V_{p}=\sqrt{(\lambda+2 \mu) / \rho}$ and $V_{s}=\sqrt{\mu / \rho}$. This problem is closed 
with boundary and initial conditions. For instance, in an infinite medium, the Sommerfeld radiation conditions state:

$$
\lim _{r \rightarrow \infty} r\left(\frac{\partial \mathbf{u}}{\partial r}-i k \mathbf{u}\right)=0
$$

where $r=\|\mathbf{x}\|$.

Assuming that the displacement field in Eq. (1) is expressed as a monochromatic plane wave: $\mathbf{u}=\tilde{\mathbf{u}} \exp i(\mathbf{k} \cdot \mathbf{x}-\omega t)$, where $\omega$ is the angular frequency, $\mathbf{k}$ the wave number and $\tilde{\mathbf{u}}$ the amplitude vector, we obtain in the case with no volume force:

$$
\left(\Gamma(\mathbf{k})-\rho \omega^{2} \mathbb{I}\right) \tilde{\mathbf{u}}=\mathbf{0}
$$

where $\Gamma(\mathbf{k})=(\lambda+\mu) \mathbf{k} \otimes \mathbf{k}-i(\nabla \lambda \otimes \mathbf{k}+\mathbf{k} \otimes \nabla \mu)+\left(\mu|\mathbf{k}|^{2}-i \mathbf{k} \cdot \nabla \mu\right)$ is the Christoffel tensor. The dispersion equations are defined as $\operatorname{det}\left(\Gamma(\mathbf{k})-\rho \omega^{2} \mathbb{I}\right)=0$ and indicate the $(\mathbf{k}, \omega)$ pairs that allow for the existence of non-vanishing plane wave solutions of the wave equation. The values of $\mathbf{k}$ can be real, complex or imaginary. With the convention used here (as in [22]), the real values correspond to propagative modes, pure imaginary values refer to evanescent modes, and complex values correspond to propagative modes with attenuation.

For an elastic, isotropic and 3-D homogeneous medium, the propagative solutions are such that $\omega=V_{p}|\mathbf{k}|$ or $\omega=V_{s}|\mathbf{k}|$. In the latter case, two different polarizations (direction of vector $\tilde{\mathbf{u}}$ ) exist. For most other media, dispersion relations have to be 95 computed numerically [23, 24].

\subsection{General methodology to obtain dispersion curves using time-space data}

A first approach consists in diagonalizing directly the Christoffel tensor, when available. In this paper, because the problem is too large, we rather use a methodology based on a 2D Fourier transform of numerical approximations of Eq. (1) to obtain an approximation of the pairs $(\mathbf{k}, \omega)$ that satisfy the dispersion relation [25, 10, 26]. The general methodology to construct the dispersion relation consists in computing a displacement field, $\mathbf{u}(\mathbf{x}, t)$ and performing Fourier transforms in both the time and space domains to obtain a 2D-variate function $\mathbf{H}_{\hat{\mathbf{k}}}(k, \omega)$ in a specific direction $\hat{\mathbf{k}}=\mathbf{k} / k$ with $k=\|\mathbf{k}\|:$

$$
\mathbf{H}_{\hat{\mathbf{k}}}(k, \omega)=\int_{-\infty}^{+\infty} \int_{-\infty}^{+\infty} \mathbf{u}(\mathbf{x}, t) e^{-i(\mathbf{k} \cdot \mathbf{x}+\omega t)} \mathrm{d} x_{k} \mathrm{~d} t
$$


Note that $x_{k}=\mathbf{x} \cdot \hat{\mathbf{k}}$ represents the coordinate of the space position along the selected direction. The locations in $(k, \omega)$ where the amplitude $\left\|\mathbf{H}_{\hat{\mathbf{k}}}(k, \omega)\right\|$ is large match the dispersion equation [25]. Note that advantage is taken of the property of the method to resolve multimodal signals (more than one wave mode in the same time signal).

In order to compute an approximation of Eq. (4), we use $\mathbf{u}(\mathbf{x}, t)$ estimated in positions $\left\{\mathbf{x}_{m}\right\}_{1 \leq m \leq M}$ and aligned along direction $\hat{\mathbf{k}}$, and record the quantity of interest in those sensors at different time steps $\left\{t_{n}\right\}_{1 \leq n \leq N}$. Assuming that the sensors are uniformly distributed along a segment of length $L$, and that the time instants are uniformly distributed in an interval of length $T$, we obtain the following map:

$$
H_{p q}=\left|\sum_{m=1}^{M} \sum_{n=1}^{N} w\left(\mathbf{x}_{m} \cdot \hat{\mathbf{k}}, t_{n}\right) \mathbf{u}\left(\mathbf{x}_{m}, t_{n}\right) e^{-2 \pi i\left(\frac{p m}{M}+\frac{q n}{N}\right)}\right|=\left|\mathrm{DFT}_{2 \mathrm{D}}\left[w\left(\mathbf{x}_{m} \cdot \hat{\mathbf{k}}, t_{n}\right) \mathbf{u}\left(\mathbf{x}_{m}, t_{n}\right)\right]\right|
$$

where $p=\mathbf{k} L$ is the normalized wave-number, $q=f T$ is the normalized frequency and $\mathrm{DFT}_{2 \mathrm{D}}$ represents the 2D Discrete Fourier Transform. To avoid the presence of side lobes and reduce leakage, a bi-dimensional Tukey (tapered cosine) window function $w\left(x_{k}, t\right)$ was applied [27, 28].

In many examples described in this paper, the maps cited above are computed as averages of such functions using $P$ lines of sensors, and $N_{\mathrm{MC}}$ realizations of the random medium (to be discussed later). In that case, the formulas above are computed for each line and each realization independently, and the average is computed dividing by $P \times N_{\mathrm{MC}}$. Also note that the source used for the construction of the maps has a very flat spectrum so the solution $\mathbf{u}(x, t)$ can be used directly. However, all results will be de-convolved (actually normalized by the source in the Fourier space of frequencies) before computing the maps.

\subsection{Spectral Element solver}

In this paper, Eq. (1) is approximated with the Spectral Element Method (SEM). The SEM is a high-order Finite Element Method that uses Gauss-Lobatto-Legendre (GLL) quadrature rule and Lagrange polynomials based on the nodes of that same quadrature. This ensures that the mass matrix is diagonal and allows to use explicit time integration schemes and very efficient parallelization (for a complete description 
of the method, see for instance [29], as well as the original papers [30, 31]). The space discretization of the variational form of the wave propagation Eq. (1) gives:

$$
\mathbf{M} \dot{\mathbf{V}}=\mathbf{F}^{\mathrm{ext}}-\mathbf{F}^{\mathrm{int}}(\mathbf{U}, \mathbf{V})
$$

where $\mathbf{U}$ and $\mathbf{V}$ are vectors containing the components of the displacement and velocity at the nodes, $\mathbf{M}$ is the (diagonal) mass matrix, and the vectors $\mathbf{F}^{\text {ext }}$ and $\mathbf{F}^{\text {int }}$ are vectors of external loads and internal forces. Using an explicit second-order finite-difference scheme in time leads to:

$$
\begin{gathered}
\frac{1}{\Delta t} \mathbf{M}\left[\mathbf{V}_{\mathbf{n}+\mathbf{1}}-\mathbf{V}_{\mathbf{n}}\right]=\mathbf{F}_{\mathbf{n}+\mathbf{1} / \mathbf{2}}^{\mathbf{e x t}}-\mathbf{F}^{\mathrm{int}}\left(\mathbf{U}_{\mathbf{n}+\mathbf{1} / \mathbf{2}}, \mathbf{V}_{\mathbf{n}+\mathbf{1} / \mathbf{2}}\right), \\
\mathbf{U}_{\mathbf{n}+\mathbf{1}}=\mathbf{U}_{\mathbf{n}}+\frac{1}{2 \Delta t}\left[\mathbf{V}_{\mathbf{n}}+\mathbf{V}_{\mathbf{n}+\mathbf{1}}\right] \\
\mathbf{A}_{\mathbf{n}+\mathbf{1}}=\frac{1}{\Delta t}\left[\mathbf{V}_{\mathbf{n}+\mathbf{1}}-\mathbf{V}_{\mathbf{n}}\right]
\end{gathered}
$$

where $\mathbf{U}_{\mathbf{n}+\mathbf{1} / \mathbf{2}}=\left(\mathbf{U}_{\mathbf{n}+\mathbf{1}}+\mathbf{U}_{\mathbf{n}}\right) / 2$ and $\mathbf{F}_{\mathbf{n}+\mathbf{1} / \mathbf{2}}^{\text {ext }}=\left(\mathbf{F}_{\mathbf{n}+\mathbf{1}}^{\text {ext }}+\mathbf{F}_{\mathbf{n}}^{\text {ext }}\right) / 2$. Even though the stability condition requires to use very small time steps [29, 32, 33], the construction of the solution at each time step is very cheap because the inversion of the mass matrix is instantaneous. Our implementation uses hexahedral non-structured meshes and Perfectly Matched Layers (PML) to account for infinite domains. PMLs are fictitious layers of materials added at the outer edges of the computational domain to absorb outgoing waves with as little reflection as possible at the interface [34, 35, 36, 37]. All simulations presented in this paper ran at Moulon Mesocentre facility in France, jointly managed by CentraleSupélec and ENS Paris-Saclay. The cluster uses Intel Xeon processors E5-2670 v3 @ 2.30 GHz (Haswell).

\subsection{Verification of the methodology in the infinite isotropic case}

This last part of the section is devoted to a verification of the methodology introduced in Section 2.2 in the isotropic case, for which the dispersion curve is known. Indeed, as indicated in Section 2.1, the dispersion curve in infinite isotropic media is composed of two lines, with equations $\omega=V_{p}|\mathbf{k}|$ and $\omega=V_{s}|\mathbf{k}|$. This last section 


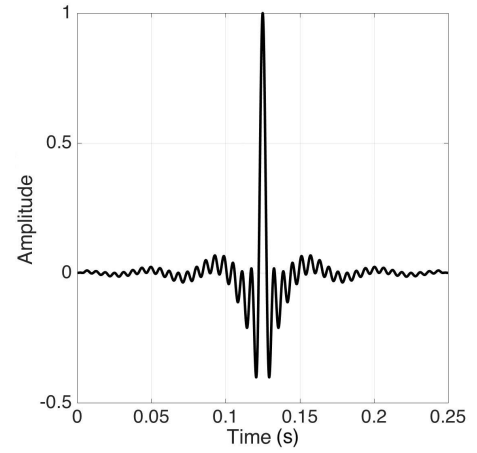

(a)

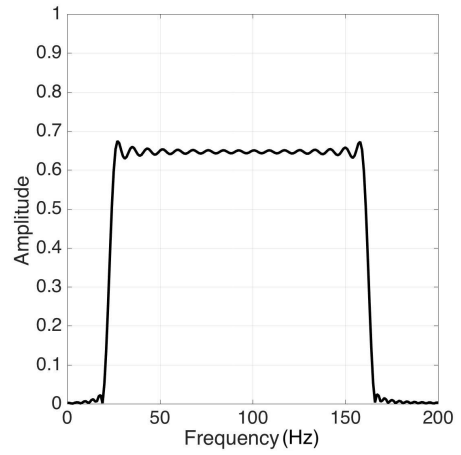

(b)

Figure 1: Time history of the amplitude of the point source (a) and frequency spectrum (b) for the verification example in Section 2.4

therefore aims at showing that the proposed numerical methodology indeed reveals these two lines in the isotropic case.

An isotropic solid is considered, whose mechanical properties are $V_{p}=200 \mathrm{~m} / \mathrm{s}$, $V_{s}=100 \mathrm{~m} / \mathrm{s}$, and $\rho=1450 \mathrm{~kg} / \mathrm{m}^{3}$. The numerical model is a cube of side $L=5 \lambda_{\max }=$ $50 \mathrm{~m}$, where $\lambda_{\max }=10 \mathrm{~m}$ is approximately the longest wavelength in the model (see below the loading frequency range). PMLs are positioned on all sides in order to mimic an infinite medium. A point load is positioned at $[1,1,1] \lambda_{\max }$, with polarization along the $x$ direction. To create a source with almost flat spectrum, the load amplitude is given by an Ormsby function:

$$
\begin{aligned}
A(t)=\left[\frac{\left(\pi f_{4} \operatorname{sinc}\left(\pi f_{4} t\right)\right)^{2}}{\pi\left(f_{4}-f_{3}\right)}-\frac{\left(\pi f_{3} \operatorname{sinc}\left(\pi f_{3} t\right)\right)^{2}}{\pi\left(f_{4}-f_{3}\right)}\right]- & \\
& {\left[\frac{\left(\pi f_{2} \operatorname{sinc}\left(\pi f_{2} t\right)\right)^{2}}{\pi\left(f_{2}-f_{1}\right)}-\frac{\left(\pi f_{1} \operatorname{sinc}\left(\pi f_{1} t\right)\right)^{2}}{\pi\left(f_{2}-f_{1}\right)}\right], }
\end{aligned}
$$

with $f_{1}=20 \mathrm{~Hz}, f_{2}=25 \mathrm{~Hz}, f_{3}=160 \mathrm{~Hz}$, and $f_{4}=165 \mathrm{~Hz}$. This function displays a quasi-plane spectrum between frequencies $f_{2}$ and $f_{3}$, with a low-cut frequency $f_{1}$ and a high-cut frequency $f_{4}$. Fig. 1 displays the time history and frequency spectrum of the loading.

The mesh is composed of hexahedral elements of edge $\lambda_{\min } / 2=0.3125 \mathrm{~m}$. Each element uses 4th-order Lagrange polynomials in each direction, for a total of 375 de- 


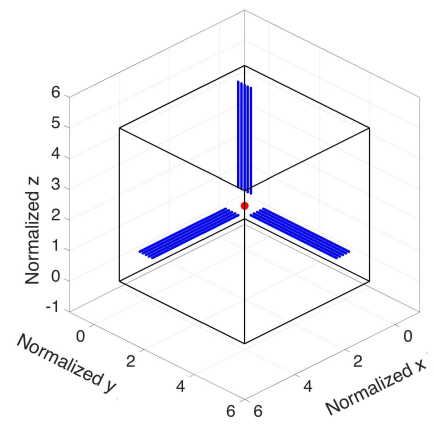

Figure 2: General setting of the verification example in Sec. 2.4 Monitors positions (5 blue lines along each direction of space), source position (red dot). The exterior cube represents the numerical domain without the PMLs.

grees of freedom per element and 4'096'000 elements in the mesh. On all sides, 5 layers of PMLs are added, with a total thickness $0.255 \lambda_{\max }$. The damping evolution in the PMLs is parameterized by a polynomial degree $p=3$ and amplitude $\alpha=9.87$ [38]. The model was run on 144 processors, and required around $140 \mathrm{~h}$ of CPU time.

The methodology proposed in Sec. 2.2 was then applied. In order to evaluate Eq. (5), sensors are placed along different lines and record the displacement field. Three groups of 5 lines of sensors were placed aligned with the cube axes. Each line has a total length of $3.5 \lambda_{\max }$, with equally spaced sensor by $\lambda_{\min } / 10$. Finally, the distance between two parallel lines is $\lambda_{\min } / 2$. The general setting of sensors is represented on Fig. 2

Fig. 3 presents the time history in the numerical domain. As the PML domain is fictitious, its local solution is not relevant to the mechanical problem and is not displayed in the images. There is no apparent reflection coming out of the PML, which indicates that it is accurately playing its role of absorbing outgoing waves. We can clearly see the $\mathrm{P}$ and $\mathrm{S}$ waves with different velocities. The $\mathrm{P}$ wave, faster then the $\mathrm{S}$ wave, presents large amplitudes along the $x$-axis and vanishing amplitude along the $y$ and $z$ axes. This is due to the polarization of the source along the $\mathrm{x}$-axis. As the $\mathrm{P}$-wave is a pressure wave, it is indeed strongly excited by the source. On the other hand, such 155 a source does not generate pressure perpendicular to its polarization. For the S-wave, 


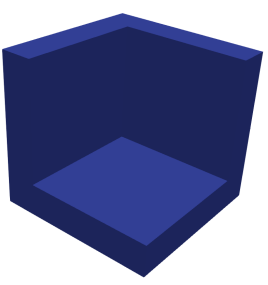

(a)

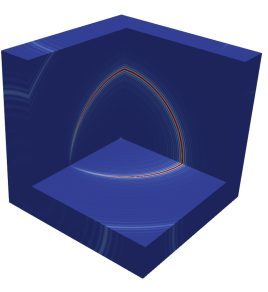

(d)

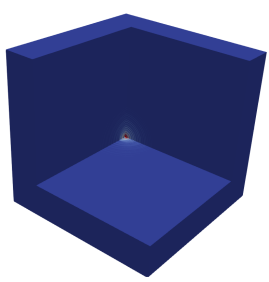

(b)

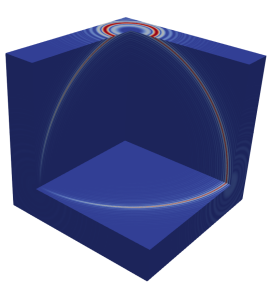

(e)
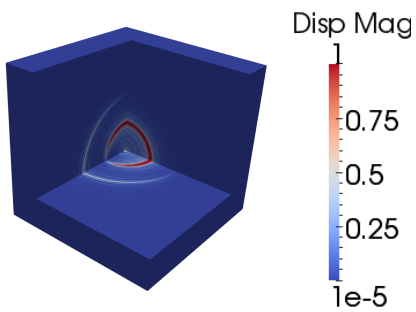

(c)
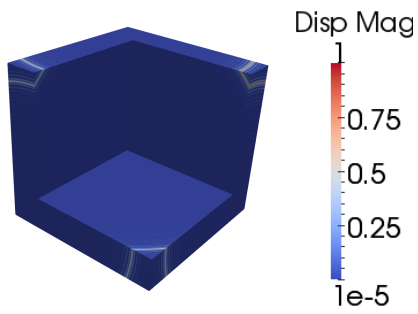

(f)

Figure 3: Normalized displacement fields in an infinite elastic isotropic homogeneous model at times: (a) $t=$ $0.000 \mathrm{~s}$; (b) $t=0.141 \mathrm{~s}$; (c) $t=0.282 \mathrm{~s}$; (d) $t=0.423 \mathrm{~s}$; (e) $t=0.564 \mathrm{~s}$; (f) $t=0.705 \mathrm{~s}$.

the opposite is logically observed, with large amplitudes along the $y$ and $z$ axes and vanishing amplitude along the $x$-axis. The displacement field used in Fig. 3 is obtained by normalizing with the maximum displacement at $0.25 \mathrm{~s}$.

Fig. 4 displays the dispersion curves obtained through the methodology described in Section 2.2 using displacements obtained with the above setting. The colors indicate the values of $H_{p q}$ in decibels. There is clearly an excellent agreement between the values obtained using the proposed methodology and the analytical values of the dispersion curves. Because of the polarization of the source, the P-wave dispersion curve is only observed in the sensors aligned along the $x$-axis, while the $\mathrm{S}$-wave dispersion curves are observed in the other two directions.

\section{Dynamic behavior of a ballasted railway track}

In this section, we now present the construction of the dispersion curves for a ballasted railway track. First, the setup of the model is described. Then, we provide some 


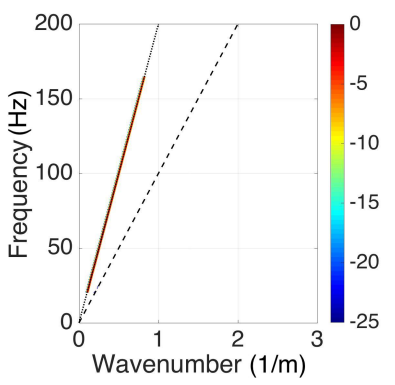

(a)

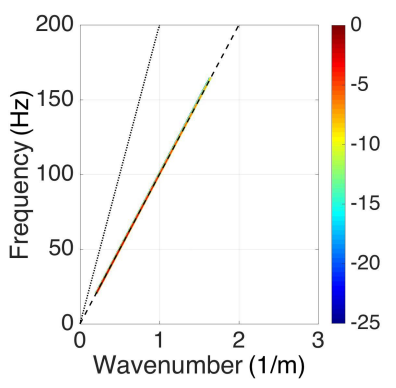

(b)

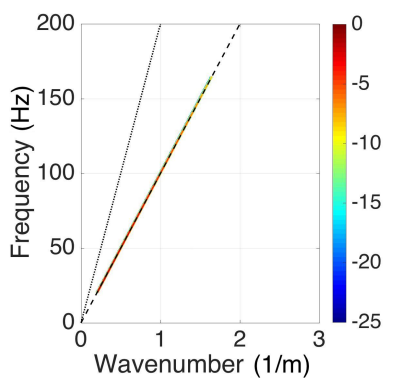

(c)

Figure 4: Dispersion curves for an infinite homogeneous media along axes x (figure a), y (figure b) and z (figure c). The color scale is the amplitude of $H_{p q}$ evaluated in Eq. [5, limited to values above $-25 \mathrm{~dB}$. The dotted black line is the analytical solution for the pressure waves dispersion relation. The dashed black line is the analytical solution for the shear wave.

snapshots of the solution in time and analyze them. Finally, the construction of the dispersion curve itself is presented and discussed.

\subsection{Randomly heterogeneous continuum model of a ballasted railway track}

The ballasted railway track is modeled over a length of $84.375 \mathrm{~m}$ as an elastic halfspace topped by a wedge-shaped structure, whose geometry is invariant along one direction (see Fig. 5). The structure represents the ballast while the elastic half-space represents the soil that supports it. The soil is modeled as a bounded box with dimensions $3 \times 8 \times 84.375 \mathrm{~m}^{3}$ and with the following properties: $V_{s}=180 \mathrm{~m} / \mathrm{s}, V_{p}=1100 \mathrm{~m} / \mathrm{s}$, and $\rho=1900 \mathrm{~kg} / \mathrm{m}^{3}$ (see further down for the description of the ballast). The box of soil is surrounded on five sides by a layer of PMLs, that allow the wave to exit the box with little reflection, mimicking an infinite medium. The total thickness of the PML layer is $2.45 \mathrm{~m}$, and the damping evolution in the PMLs is parameterized by a polynomial degree $p=3$ and amplitude $\alpha=9.87$. The mesh, shown as insert in Fig. 5 is discretized by 2.4 millions of non-structured elements, with 375 degrees of freedoms (DOFs) in each element, which amounts to a total of $\approx 160$ millions DOFs in the entire model. Note that this model was already used in [39], although with a different mesh.

A series of point forces polarized in the $[1 ; 1 ; 1]$ direction, are positioned at the top of the ballast layer, $9 \mathrm{~m}$ away from the border of the model in a non-symmetric arrange- 


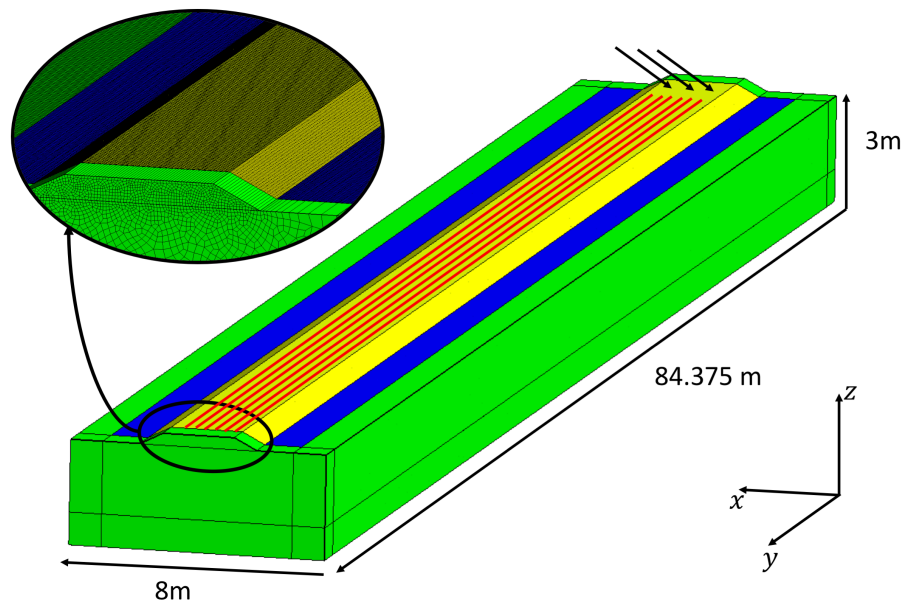

Figure 5: Geometry, loading (black arrows), and sensors (lines of red dots) for the model. The insert displays the mesh. The ballast appears in yellow, the soil in blue, and the PML region in green.

ment in the transversal section. These sources are expected to excite both shear and longitudinal waves in the model. Their amplitudes are given by an Ormsby function with a plane spectrum between 10 and $400 \mathrm{~Hz}\left(f_{1}=8 \mathrm{~Hz}, f_{2}=10 \mathrm{~Hz}, f_{3}=400 \mathrm{~Hz}\right.$, and $f_{4}=410 \mathrm{~Hz}$ in Eq. (10). The displacement is recorded in sensors located along six parallel lines at the top of the ballast layer and aligned with the axis of the track (see Fig. 5). The lines are 72 meters-long and the sensors are separated by $0.0732 \mathrm{~m}$ $\approx \lambda_{\min } / 10$. This sampling is sufficient to be able to compute the Fourier transform in space accurately.

The ballast is modeled as a continuum-based randomly-fluctuating elastic medium, as introduced in [4]. Following the polar decomposition of the strain-stress tensor [40]), the density, $\rho$, and the shear, $\mu$, and bulk moduli, $\kappa$, are taken as fully correlated stationary random fields indexed in space. The first-order marginal density for each of these random fields is taken as gamma law with averages $\rho=1570 \mathrm{~kg} / \mathrm{m}^{3}, \underline{\kappa}=$ $179.60 \mathrm{MN} / \mathrm{m}^{2}$ and $\underline{\mu}=35.32 \mathrm{MN} / \mathrm{m}^{2}$, and standard deviations $\sigma_{\rho}=3925 \mathrm{~kg} / \mathrm{m}^{3}$, $\sigma_{\kappa}=449.02 \mathrm{MN} / \mathrm{m}^{2}$ and $\sigma_{\mu}=88.31 \mathrm{MN} / \mathrm{m}^{2}$. This corresponds to a ballast layer with (arithmetic) average velocities $V_{s}=150 \mathrm{~m} / \mathrm{s}$ and $V_{p}=380 \mathrm{~m} / \mathrm{s}$ [39]. In such heterogeneous setting, the apparent velocities of the homogenized waves are expected to 


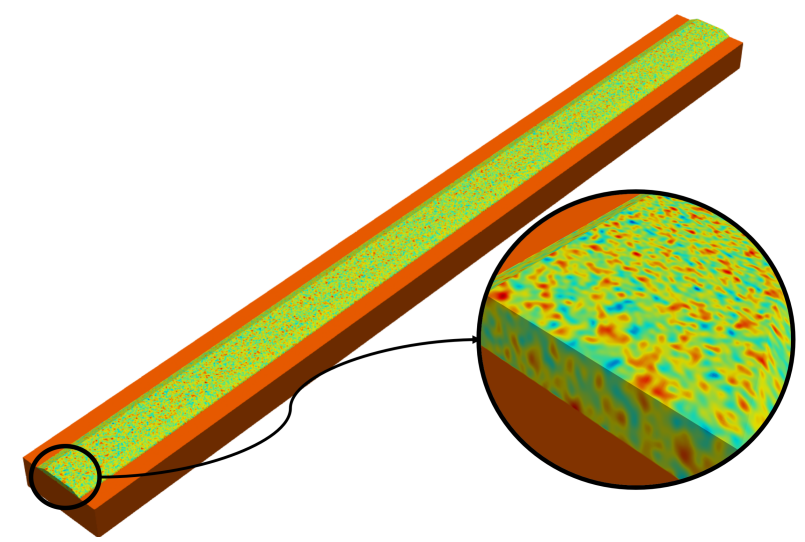

Figure 6: One realization of the density field in a ballasted railway track (PML not represented).

be close to the harmonic averages, which are here approximately $\bar{V}_{p}=280 \mathrm{~m} / \mathrm{s}$ and $\bar{V}_{s}=110 \mathrm{~m} / \mathrm{s}$ for the pressure and shear waves, respectively. Note that the standard deviations are equal to 2.5 times the averages, so that the fluctuations are very large. The correlation model [41, 42, 43] corresponds to that of a dense packing of impenetrable spheres of uniform diameter $d=3.9 \mathrm{~cm}$ in void, with volume ratio $\phi=0.583$. More details, including identification and comparison with simulations with the discrete element method, can be found in [4]. Realizations of the random fields can be obtained using a spectral representation method, specifically tailored for large scale parallel applications [44], and made available as an open-source software [45]. One sample of the random field is provided in Fig. 6

\subsection{Analysis of the wave patterns}

Fig. 7 displays snapshots at different times of the normalized displacement field in the ballasted railway track, with a normalization similar to that of Fig. 3, that is to say a normalization by the maximum displacement at time $t=0.25 \mathrm{~s}$. The first observation is that a large part of the energy seems to remain localized within the vicinity of the source. This is not what is expected from a simulation in a homogeneous domain [46, 4], where waves should propagate non-dispersively with the velocity of a Rayleigh wave. In a setting including a homogeneous ballast layer, the situation would be more complex, and probably involve dispersive waves in the waveguide [46]. How- 
ever, the fact that part of the energy remains localized close to the source is a specific feature of the heterogeneous model of the ballast. On the snapshots at longer times (at $t=0.585 \mathrm{~s}$ for instance), there seems to be a separation between waves with longer wavelengths that actually propagate away from the source, and shorter wavelengths that remain localized around the source. This effect therefore seems to be dependent on frequency. A possible explanation for this phenomenon, that seems to be stationary in time, and strongly selective in frequency, is Anderson localization [16, 5, 47]. First discovered in 1958 by P. W. Anderson [16] for the Schrödinger equation, it explains why pure metals are conductors while strongly heterogeneous metals - that is to say metals with high density of defects in the cristalline structure - are insulators. Later on, it was acknowledged as ubiquitous to all classical wave equations (acoustic, electromagnetic, elastic) [17], and explains that energy cannot propagate away from the source in strongly heterogeneous non-periodic media. Anderson received the Nobel Prize in physics in 1977 for that discovery. The absence of propagative features can be observed in the dispersion relation through the absence of $(\omega, k)$ pairs in the considered frequency range, that is to say a disappearance of the dispersion curve. Such a disappearance will be discussed in the next section.

\subsection{Construction of the dispersion curves}

We now turn to the construction of the dispersion curves for our model, using the methodology presented in Sec. 2.2 and based on displacement field acquired along the sensors lines indicated in Fig. 5. The maps $H_{p q}$ are obtained for each of the 6 lines independently and for 5 different random samples of the ballast layer. The final map is obtained as an average over these 30 individual maps and is plotted in Fig. 8

In this figure, three main frequency regimes are identified. Above about $100 \mathrm{~Hz}$, the figure appears blank, which means that no energy propagates. Of course, this does not mean that no energy exists in that frequency range, since the source contains frequencies up to $400 \mathrm{~Hz}$. It just does not propagate along the axis of the track (the direction that is being monitored here), which is consistent with the observations at short wavelengths made on Fig. 7 in the previous section. Energy in that high-frequency range actually remains mostly localized in the vicinity of the source, as predicted by Ander- 


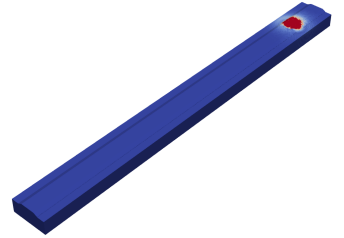

(a)

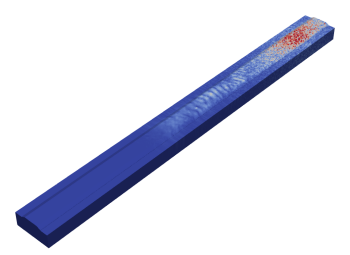

(d)

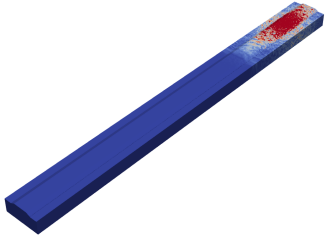

(b)

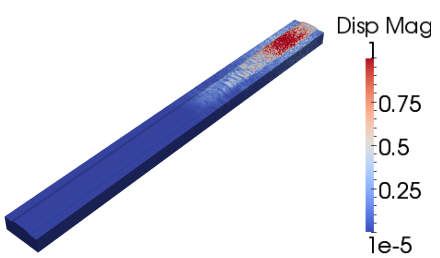

(c)

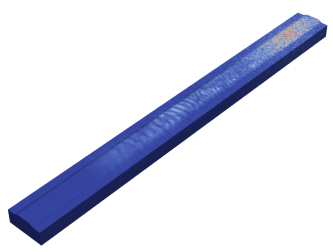

(e)

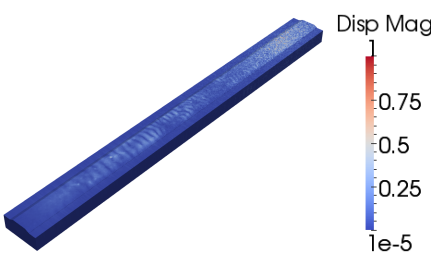

(f)

Figure 7: Normalized displacement fields in the ballasted railway track at times: (a) $t=0.135 \mathrm{~s}$; (b) $t=$ $0.225 \mathrm{~s}$; (c) $t=0.315 \mathrm{~s}$; (d) $t=0.405 \mathrm{~s}$; (e) $t=0.495 \mathrm{~s}$; (f) $t=0.585 \mathrm{~s}$.

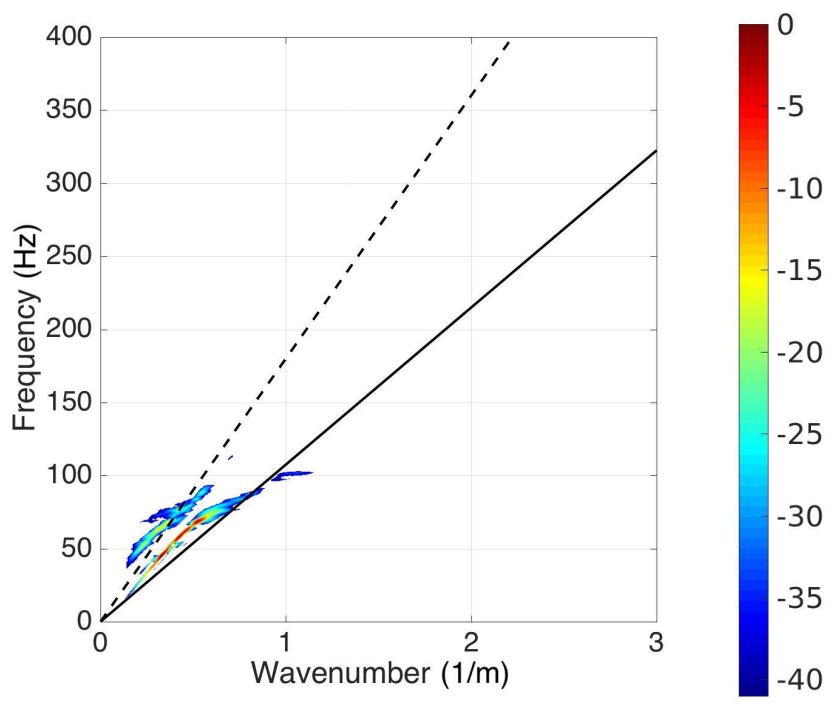

Figure 8: Dispersion curve for a heterogeneous ballast resting on a homogeneous soil. Dispersion relations for the homogenized ballast shear wave (black solid line) and soil shear wave (black dashed line) are also indicated. The color scale is the amplitude of the average $H_{p q}$ in Eq. 5, averaged over 6 sensor lines and 5 random realizations of the ballast layer. The color scale is limited at $-40 \mathrm{~dB}$. 


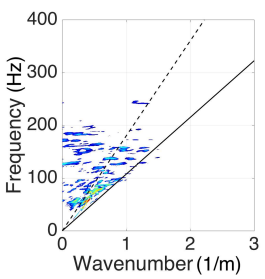

(a)

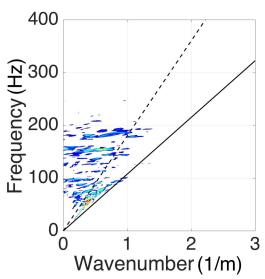

(b)

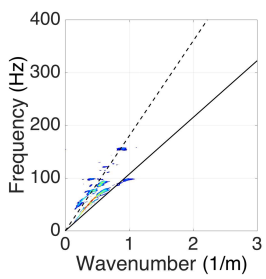

(c)

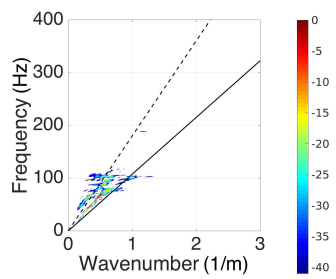

(d)

Figure 9: Dispersion curves for individual maps: for two different lines in the same realization of the ballast layer (no average, figures (a) and (b)), and for two different realizations of the ballast layer (averaged over the 6 lines for that realization, figures (c) and (d)). Dispersion relations for the homogenized ballast shear wave (black solid line) and soil shear wave (black dashed line) are also indicated. The color scale is limited at $-40 \mathrm{~dB}$.

son localization. Below about $50 \mathrm{~Hz}$, there seems to be mainly an S-wave (or more probably a Rayleigh wave, whose velocity is slightly smaller) propagating in the soil. At such long wavelengths (compared to the thickness of the ballast), the wave does not really interact with the ballast layer and propagates as if in a homogeneous soil without ballast. Between about $50 \mathrm{~Hz}$ and $100 \mathrm{~Hz}$, the soil S/Rayleigh wave starts interacting with the ballast, and its apparent velocity is strongly reduced, approximating that of the ballast layer. A P wave also seems to appear in the ballast (faster than in the soil), bending rapidly with increasing frequency. This general behavior is consistent with experimental observations in unconsolidated granular packings [10].

To get an idea of the fluctuations among the different independent maps (before averaging), the maps corresponding to two different lines for the first realization of the random ballast are plotted in Fig. 9, along with the maps corresponding to the average over the 6 lines for two different random realizations of the ballast layer. The few maps presented here are representative of the entire set. Although the average on the lines and the realizations reduces noise, the main features discussed above are present in each and every individual curve. These features are therefore not a chance effect obtained from averaging but really a characteristic behavior of ballasted railway tracks.

Figs. 8 and 9 clearly state that no wave can propagate along the direction of the 
track with frequency above $100 \mathrm{~Hz}$ (wave number above $1 \mathrm{~m}-1$ ), and that waves with frequency between $50 \mathrm{~Hz}$ and $100 \mathrm{~Hz}$ propagate more slowly than wave below $50 \mathrm{~Hz}$. This of course reads more precisely in the dispersion curves but was already qualitatively seen at later times on Fig. 7. What cannot be read on the dispersion curves is what happens to the energy of these higher frequency waves since there is no damping in this model. Observation of Fig. 7 indicates that at least part of this energy remains in the vicinity of the source. Although it is widely known that high frequency waves get dissipated within the ballast, the most novel finding here is a proposal for the mechanism that leads to the trapping of high frequency waves in that ballast: Anderson localization. Note that Anderson localization does not involve any non-linear mechanism, so that it is advocated that energy becomes trapped in the ballast layer by heterogeneity, before it is eventually dissipated (in particular by friction between grains, which is not modeled here).

\section{Strong localization analysis}

In the previous section, appearance of Anderson localization in ballasted railway tracks was hinted at, with its classical stationary character and a clear frequency dependence. However, there is a possibility that this localization might be some type of guided wave effect, due to the particular geometry of the ballast. This section aims at removing the ambiguity by showing that the same feature would be present in an infinite domain, composed only of ballast material.

\subsection{Description of the numerical model}

To remove the effect of the ballast geometry and its interface with the underlying soil, we consider an infinite domain, as in Section 2.4 This domain is modeled as a cube of edge $10.98 \mathrm{~m}$, with PMLs all around. The mesh is discretized with a uniform grid of $\approx 7.2$ millions cube elements with edge $0.06 \mathrm{~m}$. Polynomials of 4 th order are used, inducing close to 1.4 billion DOFs in the entire model. The PML uses 3 layers of elements with power degree 2, amplitude 3.71 and total thickness of the PML equal to $1.69 \mathrm{~m}$. A point force is positioned at $[0.66,0.66,0.66] \mathrm{m}$, and polarized 
in the $x$ direction. The time history of that source is that of Eq. (10) with a plane

\subsection{Dispersion curve for an infinite medium of ballast material}

Eq. (5) was evaluated as an average over the 91 lines monitored for both the homogeneous and heterogeneous cases and Fig. 13 presents the obtained maps $H_{p q}$. The 


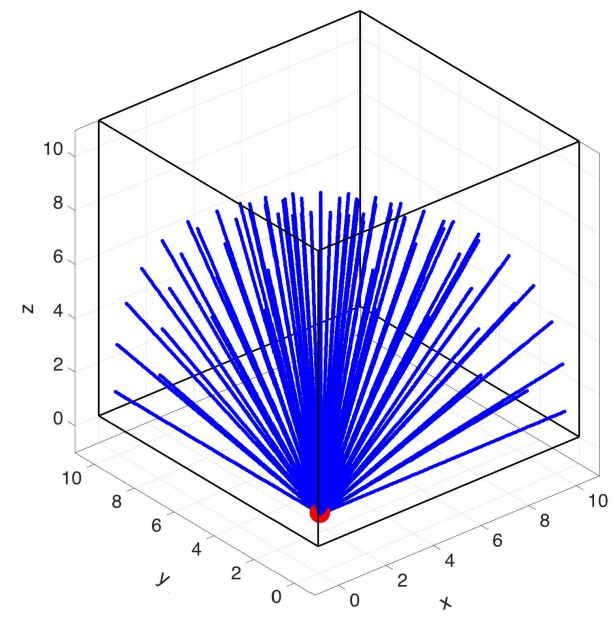

Figure 10: Position of the monitors (blue line) and the source (red point).

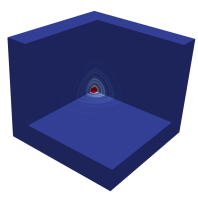

(a)

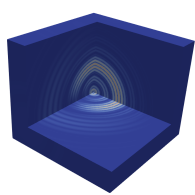

(b)

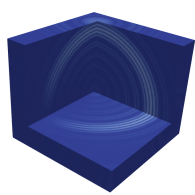

(c)

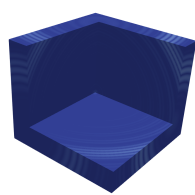

(d)

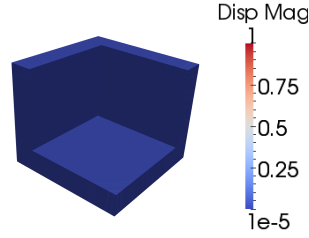

(e)

Figure 11: Normalized amplitude of the displacement field in a homogeneous infinite medium at times: (a) $t=0.094 \mathrm{~s}$; (b) $t=0.188 \mathrm{~s}$; (c) $t=0.282 \mathrm{~s}$; (d) $t=0.329 \mathrm{~s}$; (e) $t=0.470 \mathrm{~s}$.

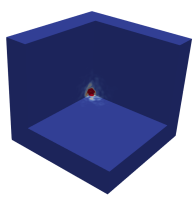

(a)

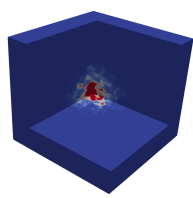

(b)

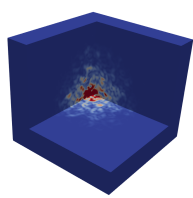

(c)

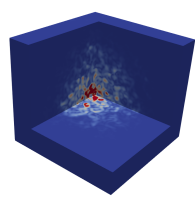

(d)

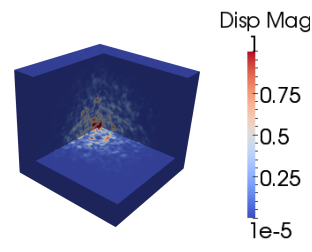

(e)

Figure 12: Normalized amplitude of the displacement field in a heterogeneous infinite medium at times: (a) $t=0.094 \mathrm{~s}$; (b) $t=0.188 \mathrm{~s}$; (c) $t=0.282 \mathrm{~s}$; (d) $t=0.329 \mathrm{~s}$; (e) $t=0.470 \mathrm{~s}$. 
reference value for the decibel scale was picked as the maximum value from the homogeneous case for both maps. As expected, a good agreement is obtained in the homogeneous case with both the analytical shear wave and pressure wave modes. The amplitude of the dispersion curve for the $\mathrm{P}$ wave appears smaller because of the average among 91 lines who are mostly oriented to record S-polarized displacements. Indeed, only 1 line is perfectly oriented to record the $\mathrm{P}$ wave (along the axis of polarization of the source), while 10 lines are perfectly oriented to record S waves (in the plane going through the source and normal to its polarization).

Turning to the heterogeneous model, the filtering effect at $100 \mathrm{~Hz}$ is clearly observed as in the ballasted railway track of the previous section. This shows that it is indeed an intrinsic property of the heterogeneous material, and not a wave guide effect due to the geometry of the ballasted track. Below $100 \mathrm{~Hz}$, however, the material behaves as a purely isotropic material, which shows that the velocity reduction observed in the ballasted railway track was indeed the consequence of the combination of ballast and soil at different wavelengths. Note that there is no apparent P-wave in this heterogeneous model for the same reason as discussed in the homogeneous case, but amplified by the heterogeneity and the weaker averaging for lines recording $\mathrm{P}$ waves.

\subsection{Characterization of Anderson localization}

In this last section, we provide additional evidence that the filtering effect above $100 \mathrm{~Hz}$ is indeed Anderson localization. Anderson localization is characterized by its strong frequency dependence, which is already apparent in the dispersion curves constructed previously. It is also characterized by the stationarity in time of the localized energy, which means that the energy remains in the vicinity of the source rather than slowly diffusing away. To illustrate this, the displacement magnitude is therefore averaged over all recording stations at the same distance from the source and plotted in Figs. 14 and 15 as a function of time and distance from the source, filtered in three frequency bands (using a second order bandpass Butterworth filter). The normalization factor for the decibel scale is the maximum displacement magnitude for complete spectra of frequencies. In the homogeneous case, the energy clearly propagates along a straight line, whose slope is controlled by the velocity of the medium. As expected 


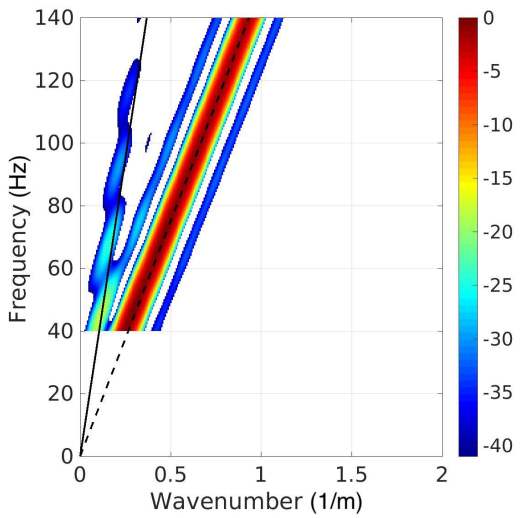

(a)

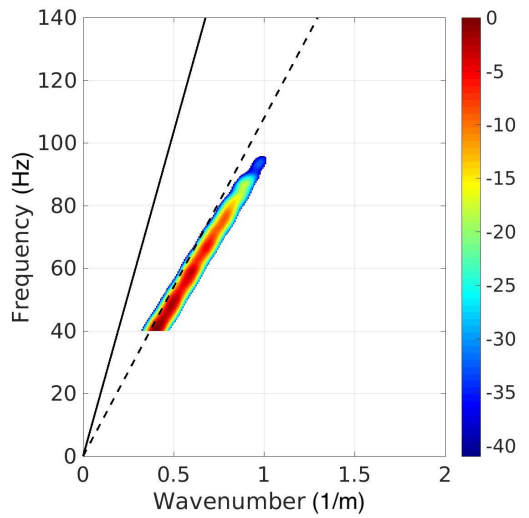

(b)

Figure 13: Dispersion curves for infinite homogeneous (figure (a)) and heterogeneous media (figure (b)). The color scale is the amplitude of $H_{p q}$ evaluated in Eq. [5], limited to values above $-40 \mathrm{~dB}$. The dotted black line is the analytical solution for the homogenized pressure waves dispersion relation. The dashed black line is the analytical solution for the homogenized shear wave.

in a non-dispersive medium, this effect is independent of frequency. On the other hand, in the heterogeneous medium, the behavior is strongly dispersive. Below $50 \mathrm{~Hz}$, an isotropic homogenized behavior is retrieved. Above $100 \mathrm{~Hz}$, the energy seems to propagate along vertical lines (zero velocity), which clearly indicates that it remains localized in time at the same distance from the source.

\section{Conclusion}

In this paper, we constructed and analyzed the dispersion curve of a ballasted railway track set on a homogeneous soil. A continuum-based randomly-fluctuating heterogeneous model was used to model the ballast. The dispersion curve of the ballasted railway track shows a tremendous influence of the heterogeneity. The behavior is dispersive with higher frequency waves propagating slower than lower frequency waves. Below $50 \mathrm{~Hz}$, the waves do not interact with the ballast layer and we retrieve the behavior of the homogeneous soil. In the frequency range between $50 \mathrm{~Hz}$ and $100 \mathrm{~Hz}$, waves seem to slow down, but still propagate at that slower pace. Above $100 \mathrm{~Hz}$, Anderson localization sets in, where the higher frequency waves remain trapped in the vicinity 


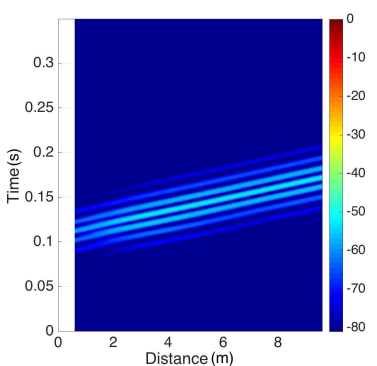

(a)

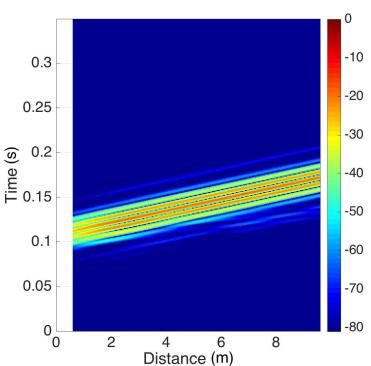

(b)

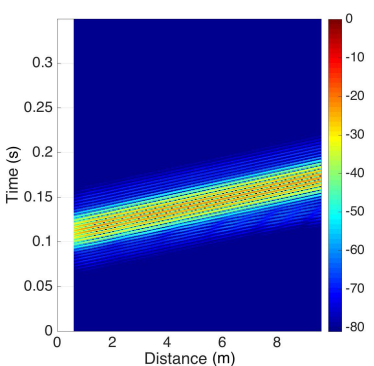

(c)

Figure 14: Displacement magnitude in an infinite homogeneous medium over different frequency ranges: (a) $0-50 \mathrm{~Hz}$; (b) $50-100 \mathrm{~Hz}$; (c) $100-150 \mathrm{~Hz}$. The color scale is the amplitude of the displacement field, limited to values above $-80 \mathrm{~dB}$.

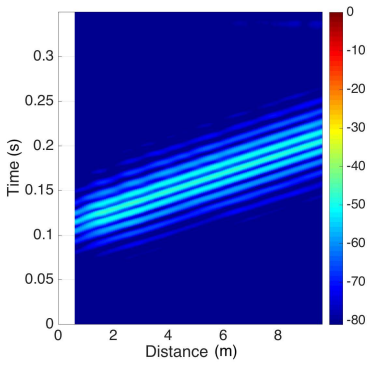

(a)

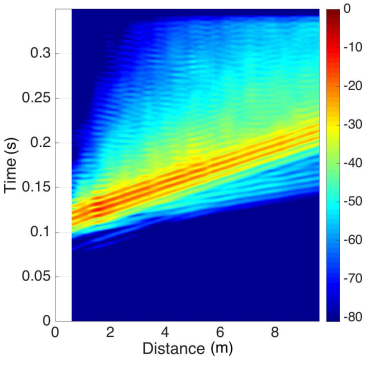

(b)

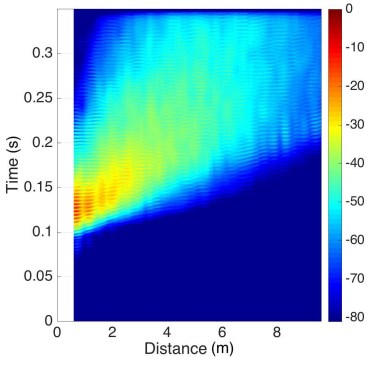

(c)

Figure 15: Displacement magnitude in an infinite heterogeneous medium over different frequency ranges: (a) $0-50 \mathrm{~Hz}$; (b) $50-100 \mathrm{~Hz}$; (c) $100-150 \mathrm{~Hz}$. The color scale is the amplitude of the displacement field, limited to values above $-80 \mathrm{~dB}$. 
of the source. Although railway engineering applications would really consider only the $[0,150] \mathrm{Hz}$ range, this paper considered a larger range to draw definite conclusions about the lack of propagation of the higher frequency waves.

Although very widely studied in the physics literature, Anderson localization has been studied in the acoustical literature mostly from the experimental angle, trying to discriminate between dissipation and localization in measurements [21]. The conclusions of this paper seem to indicate that the higher part of the energy generated by the passage of trains on a ballasted railway track remains trapped in the ballast rather than being dissipated in the surrounding soil. This is opposite to the common knowledge in the field of numerical modeling of railway-generated noise in the environment [48], although railway engineers also experience strong ballast degradation which indicates large dissipation of energy in that layer. This paper therefore proposes a physical explanation for this experimental observation, which may be a first building block towards improving ballasted railway tracks.

\section{Acknowledgements}

The spectral element software used for the simulations in this paper is developed jointly by CentraleSupélec, CEA Commissariat à l'Énergie Atomique and Institut de Physique du Globe de Paris. Within the SINAPS@ project, this development benefited from French state funding managed by the National Research Agency under program RNSR Future Investments bearing reference No. ANR-11-RSNR- 0022-04. This work was performed using HPC resources from the "Mésocentre" computing center of CentraleSupélec and École Normale Supérieure Paris-Saclay, supported by CNRS and the French Ministère de l'Enseignement Supérieur, de la Recherche, et de l'Innovation (http://mesocentre.centralesupelec.fr/).

\section{References}

\section{References}

400

[1] B. Indraratna, W. Salim, C. Rujikiatkamjorn, Advanced rail geotechnology. Ballasted track, CRC Press, Boca Raton, 2011. 
[2] M. Heckl, G. Hauck, R. Wettschureck, Structure-borne sound and vibration from rail traffic, J. Sound Vib. 193 (1) (1996) 175-184. doi:10.1006/jsvi.1996. 0257.

[3] D. P. Connolly, G. Kouroussis, O. Laghrouche, C. L. Ho, M. C. Forde, Benchmarking railway vibrations - track, vehicle, ground and building effects, Constr. Build. Mater doi:10.1016/j.conbuildmat.2014.07.042.

[4] L. de Abreu Corrêa, J. C. Quezada, R. Cottereau, S. C. d'Aguiar, C. Voivret, Randomly-fluctuating heterogeneous continuum model of a ballasted railway track, Computational Mechanics 60 (5) (2017) 845-861. doi:10.1007/ s00466-017-1446-8

[5] M. Leibig, Model for the propagation of sound in granular materials, Physical Review E 49 (2) (1994) 1647-1656. doi:10.1103/PhysRevE.49.1647.

[6] L. Rayleigh, On waves propagated along the plane surface of an elastic solid, Proceedings of the London Mathematical Society 1 (1) (1885) 4-11.

[7] H. Lamb, On waves in an elastic plate, Proceedings of the Royal Society of London A: Mathematical, Physical and Engineering Sciences 93 (648) (1917) 114128. doi:10.1098/rspa.1917.0008

[8] J. S. Langer, Lectures in the theory of pattern formation, in: J. V. J. Souletie, R. Stora (Eds.), Chance and Matter, North-Holland, Amsterdam, 1987, pp. 629711.

[9] J. Duran, Sands, powders, and grains: an introduction to the physics of granular materials, Partially Ordered Systems, Springer Science \& Business Media, NewYork, 2012.

${ }_{425}$ [10] X. Jacob, V. Aleshin, V. Tournat, P. Leclaire, W. Lauriks, V. Gusev, Acoustic probing of the jamming transition in an unconsolidated granular medium, Physical Review Letters 100 (15) (2008) 158003. 
[11] L. Bodet, X. Jacob, V. Tournat, R. Mourgues, V. Gusev, Elasticity profile of an unconsolidated granular medium inferred from guided waves: Toward acoustic monitoring of analogue models, Tectonophysics 496 (1) (2010) 99-104.

[12] A. Shukla, Dynamic photoelastic studies of wave propagation in granular media, Optics and Lasers in Engineering 14 (3) (1991) 165-184.

[13] J. Geng, D. Howell, E. Longhi, R. Behringer, G. Reydellet, L. Vanel, E. Clément, S. Luding, Footprints in sand: the response of a granular material to local perturbations, Physical Review Letters 87 (3) (2001) 035506.

[14] C.-H. Liu, S. R. Nagel, Sound and vibration in granular materials, Journal of Physics: Condensed Matter 6 (23A) (1994) A433.

[15] M. J. Buckingham, Theory of acoustic attenuation, dispersion, and pulse propagation in unconsolidated granular materials including marine sediments, The Journal of the Acoustical Society of America 102 (5) (1997) 2579-2596.

[16] P. W. Anderson, Absence of Diffusion in Certain Random Lattices, Physical Review 109 (5) (1958) 1492-1505.

[17] P. W. Anderson, The question of classical localization: a theory of white

口 paint ?, Philosophical Magazine B 52 (3) (1985) 505-509. doi:10.1080/ 13642818508240619 .

[18] R. Weaver, Anderson localization of ultrasound, Wave motion 12 (2) (1990) 129142.

[19] C.-h. Liu, S. R. Nagel, Sound in sand, Physical Review Letters 68 (15) (1992) 2301.

[20] O. I. Lobkis, R. L. Weaver, Anderson localization of ultrasound in plates: Further experimental results, The Journal of the Acoustical Society of America 124 (6) (2008) 3528-3533. 
[21] H. Hu, A. Strybulevych, J. Page, S. E. Skipetrov, B. A. van Tiggelen, Localization of ultrasound in a three-dimensional elastic network, Nature Physics 4 (12) (2008) 945-948.

[22] B. A. Auld, Acoustic fields and waves in solids, John Wiley and Sons, New-York, 1973.

[23] P. E. Lagasse, Higher order finite element analysis of topographic guides supporting elastic surface waves, The Journal of the Acoustical Society of America 53 (4) (1973) 1116-1122. doi:10.1121/1.1913432

[24] L. Knopoff, A matrix method for elastic wave problems, Bulletin of the Seismological Society of America 54 (1) (1964) 431.

[25] D. Alleyne, P. Cawley, A two-dimensional fourier transform method for the measurement of propagating multimode signals, The Journal of the Acoustical Society of America 89 (3) (1991) 1159-1168.

[26] J. O'Donovan, Micromechanics of wave propagation through granular material, Ph.D. thesis, Imperial College London (2013).

[27] F. J. Harris, On the use of windows for harmonic analysis with the discrete fourier transform, Proceedings of the IEEE 66 (1) (1978) 51-83. doi:10.1109/PROC. 1978.10837.

[28] A. Nuttall, Some windows with very good sidelobe behavior, IEEE Transactions on Acoustics, Speech, and Signal Processing 29 (1) (1981) 84-91.

[29] G. Cohen, Higher-order numerical methods for transient wave equations, Scientific Computation, Springer, Berlin Heidelberg New York, 2001.

475 [30] Y. Maday, A. T. Patera, Spectral element methods for the incompressible NavierStokes equations, in: State-of-the-art surveys on computational mechanics, no. A90-47176 21-64, American Society of Mechanical Engineers, New York, 1989, pp. 71-143. 
[31] C. Bernardi, Y. Maday, Approximations spectrales de problèmes aux limites elliptiques (Spectral approximation for elliptique boundary condition problems), Vol. 10 of Mathématiques et Applications, Springer, Berlin Heidelberg, 1992, in French.

[32] R. Sevilla, R. Cottereau, Influence of periodically fluctuating material parameters on the stability of explicit high-order spectral element methods, J. Comp. Phys. 373 (2018) 304-323. doi:10.1016/j·jcp.2018.07.002.

[33] R. Cottereau, R. Sevilla, Stability of an explicit high-order spectral element method for acoustics in heterogeneous media based on local element stability

1 criteria, Int. J. Numer. Meth. Engrg. 116 (4) (2018) 223-245. doi:10.1002/ nme.5922

[34] J.-P. Berenger, A perfectly matched layer for the absorption of electromagnetic waves, J. Chem. Phys. 114 (2) (1994) 185-200. doi:10.1006/jcph.1994. 1159 .

[35] F. Collino, P. B. Monk, Optimizing the perfectly matched layer, Comp. 口. Methods Appl. Mech. Engrg. 164 (1-2) (1998) 157-171. doi:10.1016/ S0045-7825(98)00052-8.

[36] G. Festa, J. P. Vilotte, The Newmark scheme as velocity-stress time-staggering: An efficient PML implementation for spectral element simulations of elasto1. dynamics, Geophysical Journal International 161 (3) (2005) 789-812. doi: $10.1111 / \mathrm{j} .1365-246 \mathrm{X} .2005 .02601 . \mathrm{x}$

[37] A. Modave, E. Delhez, C. Geuzaine, Optimizing perfectly matched layers in discrete contexts, Int. J. Numer. Meth. Engrg. 99 (2014) 410-437. doi:10.1002/ nme.4690

[38] M. Drozdz, Efficient finite element modelling of ultrasound waves in elastic media, Ph.D. thesis, Imperial College of Science Technology and Medicine, Londres (2008). 
[39] INNOTRACK D2.1.3, First phase on the modelling of poor quality sites, Tech. Rep. Project no. tip5 ct-2006-031415, European commission - sixth framework program (2009).

[40] Q.-A. Ta, D. Clouteau, R. Cottereau, Modeling of random anisotropic elastic media and impact on wave propagation, European Journal of Computational Mechanics/Revue Européenne de Mécanique Numérique 19 (1-3) (2010) 241-253. doi:10.3166/ejcm.19.241-253

[41] S. Torquato, G. Stell, Microstructure of two-phase random media. III. The n-point matrix probability functions for fully penetrable spheres, The Journal of Chemical Physics 82 (2) (1985) 980-987. doi:10.1063/1.445941.

[42] J. Quintanilla, Microstucture functions for random media with impenetrable particles, Phys. Rev. E 60 (1999) 5788-5794. doi:10.1103/PhysRevE.60.5788.

[43] S. Torquato, Random heterogeneous materials. Microstructure and macroscopic properties, Interdisciplinary Applied Mathematics, Springer, New York, 2001.

[44] L. de Carvalho Paludo, V. Bouvier, R. Cottereau, Scalable parallel scheme for sampling of Gaussian random fields over large domains, Int. J. Numer. Meth. Engrg. 117 (8) (2019) 845-859. doi:10.1002/nme.5981

[45] Random field library, https://github.com/cottereau/randomField, accessed: 27-01-2018.

[46] L. de Abreu Corrêa, R. Cottereau, E. Bongini, S. Costa d'Aguiar, B. Faure, C. Voivret, Impact of the heterogeneity of the ballast on the dynamical behavior of the ballast-soil system, in: Proceedings of the CM3 Conference on Computational Transport (Jyväskulä, Finland), Springer, 2016, pp. 185-205.

[47] B. P. Lawney, S. Luding, Frequency filtering in disordered granular chains, Acta mechanica 225 (8) (2014) 2385.

[48] R. Paolucci, A. Maffeis, L. Scandella, M. Stupazzini, M. Vanini, Numerical prediction of low-frequency ground vibrations induced by high-speed trains at 
Ledsgaard, Sweden, Soil Dynamics Earthquake Engr. 23 (6) (2003) 425-433.

doi:10.1016/S0267-7261(03)00061-7 